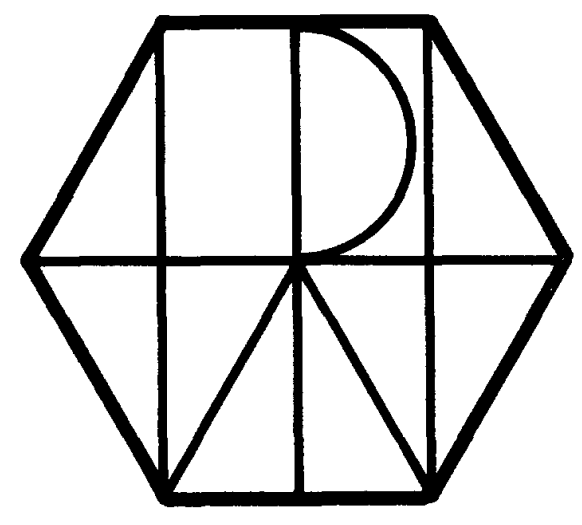

$\frac{\text { PITHA } 94 / 10}{\operatorname{sw} 9409}$

\title{
A Wiring System for Mass Production of Drift and Proportional Chambers
}

R. Bock, G. Braun, H. Eßer, K. Lübelsmeyer, W. Karpinski,

I. Pandoulas, D. Schmitz, R. Siedling, M. Wlochal

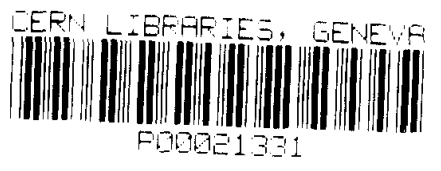

PHYSIKALISCHE INSTITUTE

RWTH AACHEN

Sommerfeldstr.

51 AACHEN, FR GERMANY 


\title{
A Wiring System for Mass Production of Drift and Proportional Chambers
}

\author{
R. Bock, G. Braur, H. EBer, K. Lübelsmeyer, W. Karpinski, \\ D. Pandoulas, D. Schmitz, R. Siedling , M. Wlochal \\ I. Physikalisches Institut, RWTH Aacheri \\ Sornmerfeldstraße 28, 52056 Aachen, Germany ${ }^{2}$
}

October 1993

\begin{abstract}
We describe the equipment and procedures developed and employed for the wiring in mass production of the forward/backward muon drift chambers of the L3 experiment. A computer-controlled machine is used to stretch sinultaneously all wires of one drift cell to the required tension. After manual insertion of the wire group into the chamber, another computer-controlled device is used to measure the tension of each wire, thus providing an immediate quality check.
\end{abstract}

\section{Introduction}

Within the framework of the international collaboration for the L3 experiment at CERN [1] the I. Physics Institute of the RWTH Aachen is participating in the development and production of the $F / B$ muon chamber system, which is part of the upgrade of the L3 detector and increases the solid angle coverage of the muon spectrometer [2] from $73 \%$ to $93 \%$ of $4 \pi$.

The F/B system consists of $6 \times 16$ trapezoidal chambers (see Fig.1), which will be mounted on the inside and the outside of the two magnet doors. In each chamber the $60 \mathrm{~mm}$ high and $1.05 \mathrm{~mm}$ wide drift cells are formed by I-beams that serve as cathodes and are insulated from the chamber body by G-10 strips. Each cell is equipped with four signal wires and five field-shaping wires (see Sec. 2). As shown in Fig. 1, each chamber consists of three layers of drift cells: two of them (called X and $W$ ) are displaced by half a cell width with respect to each other and are used to measure the azimuthal coordinate $\phi$, while the $Y$-layer measures the radial distance from the beam axis.

\footnotetext{
${ }^{2}$ Supported by the German Bundesministerium für Forschung und Technologie under contract number 056 AC16P(1)
} 


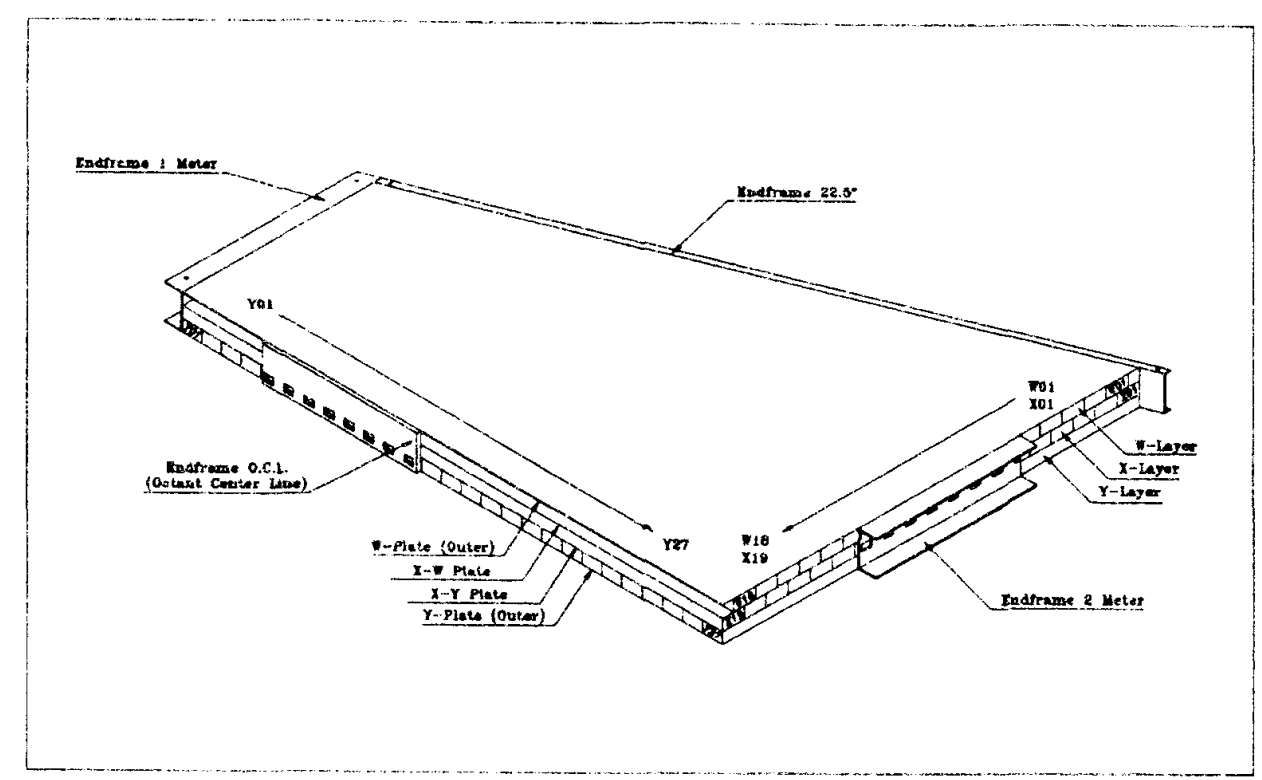

Figure 1: Cell structure of an L3 forward/backward muon chamber

\section{Choice of Wire Tension}

As already mentioned, each drift cell of the F/B muon chambers is equipped with 4 goldplated Tungsten wires (diameter $30 \mu \mathrm{m}$ ), operating as signal wires, and 5 Copper-Beryllium wires (diameter $78 \mu \mathrm{m}$ ), operating as field shaping wires. The cell length varies between approximately $470 \mathrm{~mm}$ and $2830 \mathrm{~mm}$.

No wire supports inside the chamber are used and to avoid excessive sagging of the longest wires they are stretched with a force of $1.28 \mathrm{~N}$ (signal) and $3.78 \mathrm{~N}$ (field wires). The tolerance imposed on both of these values is $\pm 4 \%$. A list of wire properties and of the wiring parameters is given in Tab. 1.

\begin{tabular}{|l|c|c|c|}
\hline parameter & symbo] & signal wires & field wires \\
\hline material & & $\begin{array}{c}\text { Tungsten-Rhenium(3\%) } \\
\text { gold plated }(0.3 \mu \mathrm{m})\end{array}$ & Copper-Beryllium \\
modulus of elasticity $\left[\frac{10^{3} \mathrm{~N}}{\mathrm{~mm}^{2}}\right]$ & $\mathrm{E}$ & 410 & 127.5 \\
density $\left[\frac{\mathrm{g}}{\mathrm{cm}^{3}}\right]$ & $\rho$ & 19.17 & 8.25 \\
linear density $\left[\frac{\mathrm{mg}}{\mathrm{m}}\right]$ & $\rho l$ & 13.45 & 39.9 \\
thermal expansion $\left[\frac{10^{-8}}{\delta}\right]$ & $\alpha$ & 4.5 & $16.8(\mathrm{Cu})$ \\
diameter $[\mu m !$ & $\mathrm{d}$ & 30 & 78 \\
tension $[N]$ & $\mathrm{F}$ & 1.28 & 3.78 \\
mass of applied weight $\lfloor g]$ & $\mathrm{m}$ & 130 & 385 \\
tolerance $(4 \%)[g]$ & & \pm 5.2 & \pm 15.4 \\
\hline
\end{tabular}

Table 1: Main wire parameters

\footnotetext{
${ }^{2}$ manufactured by LUMAMETALL, Kalrnar Sweden

${ }^{3}$ manufactured by LITTLE FACLS ALLOYS, New Jersey US
} 
The gravitational sag $s$ at a point $x$ along a wire of length 1 and linear density $\rho l$, which is stretched with a force $F$, is given by

$$
s=\frac{\rho l g x^{2}}{F} \frac{2}{2} .
$$

where $g$ is the acceleration due to gravity. With the chosen values of the tension, the sag of the long wires at $x=1 / 2$ does not exceed $100 \mu \mathrm{m}$. In the interest of simplicity and convenience during mass production (tension is applied to the wires through weights), the shorter wires are also stretched with the same force as the long ones. Consequently the sag of the shortest wires is negligible $\left[(470 / 2830)^{2} \cdot 100 \mu m \approx 3 \mu m\right]$.

The relative change in wire length due to the application of a stretching force $F$ is given by

$$
\frac{\Delta l}{l}=\frac{1}{E A} F
$$

where $A$ is the cross-sectional area and $E$ the elasticity modulus of the wire. The wire elongation resulting from application of the design tension values is given in Tab. 2.

The different thermal expansion coefficients of the wires and of the aluminium chamber body $\left(\alpha_{A l}=23.810^{-6} K^{-1}\right)$ lead to variations of the wire tension with temperature $T$. Since $\Delta l_{\text {wire }}=l_{\text {wire }} \alpha_{\text {wire }} \Delta T$ and $\Delta l_{\text {body }} \approx l_{\text {wire }} \alpha_{A l} \Delta T$, the relative change in wire length due to a ternperature change $\Delta T$ is

$$
\frac{\Delta l_{T}}{l}=\frac{\Delta l_{\text {wire }}-\Delta l_{\text {body }}}{l_{\text {wire }}}=\left(\alpha_{\text {wire }}-\alpha_{A l}\right) \Delta T .
$$

Comparing Eqs. (2) and (3) we can find the temperature change $\Delta T$ needed to get a variation of wire tension as large as the imposed tolerance of $\pm 4 \%$. From these values of $\Delta T$, also given in Tab. 2 , it is seen that for moderate temperature variations

\begin{tabular}{|c|c|c|c|c|}
\hline \multirow[b]{3}{*}{$\begin{array}{l}\text { mass of applied } \\
\text { weight }[g]\end{array}$} & \multicolumn{2}{|c|}{ signal wires } & \multicolumn{2}{|c|}{ field wires } \\
\hline & design & $\pm 4 \%$ & design & $\pm 4 \%$ \\
\hline & & & 385 & 15.4 \\
\hline$\frac{\Delta l}{l}\left[10^{-3}\right]$ (by stretching) & 4.4 & 0.18 & 6.1 & 0.25 \\
\hline$\Delta l[m m](l=2827 \mathrm{~mm})$ & 12.4 & 0.5 & 17.3 & 0.7 \\
\hline$\Delta l[\mathrm{~mm}](l=473 \mathrm{~mm})$ & 2.1 & 0.08 & 2.9 & 0.1 \\
\hline$\Delta T\left[{ }^{0} C\right]$ & & 9.2 & & 35.1 \\
\hline
\end{tabular}
significant effects car occur only for the signal wires, due to the larger difference in thermal expansion coefficients between tungsten and aluminium.

Table 2: Changes in wire length

\section{The Wiring Machine}

To allow fast and efficient stringing of the wires in the previously completely assembled bodies of the $\mathrm{F} / \mathrm{B}$ muon chambers (each chamber has 64 drift cells and thus a 
total of 576 wires), we developed and buil a computer controlled wiring machine. Fig. 2 shows a photograph and Fig. 3 a schematic diagram of the machine (in the latter only one of the aine wires of a wire group is depicted).

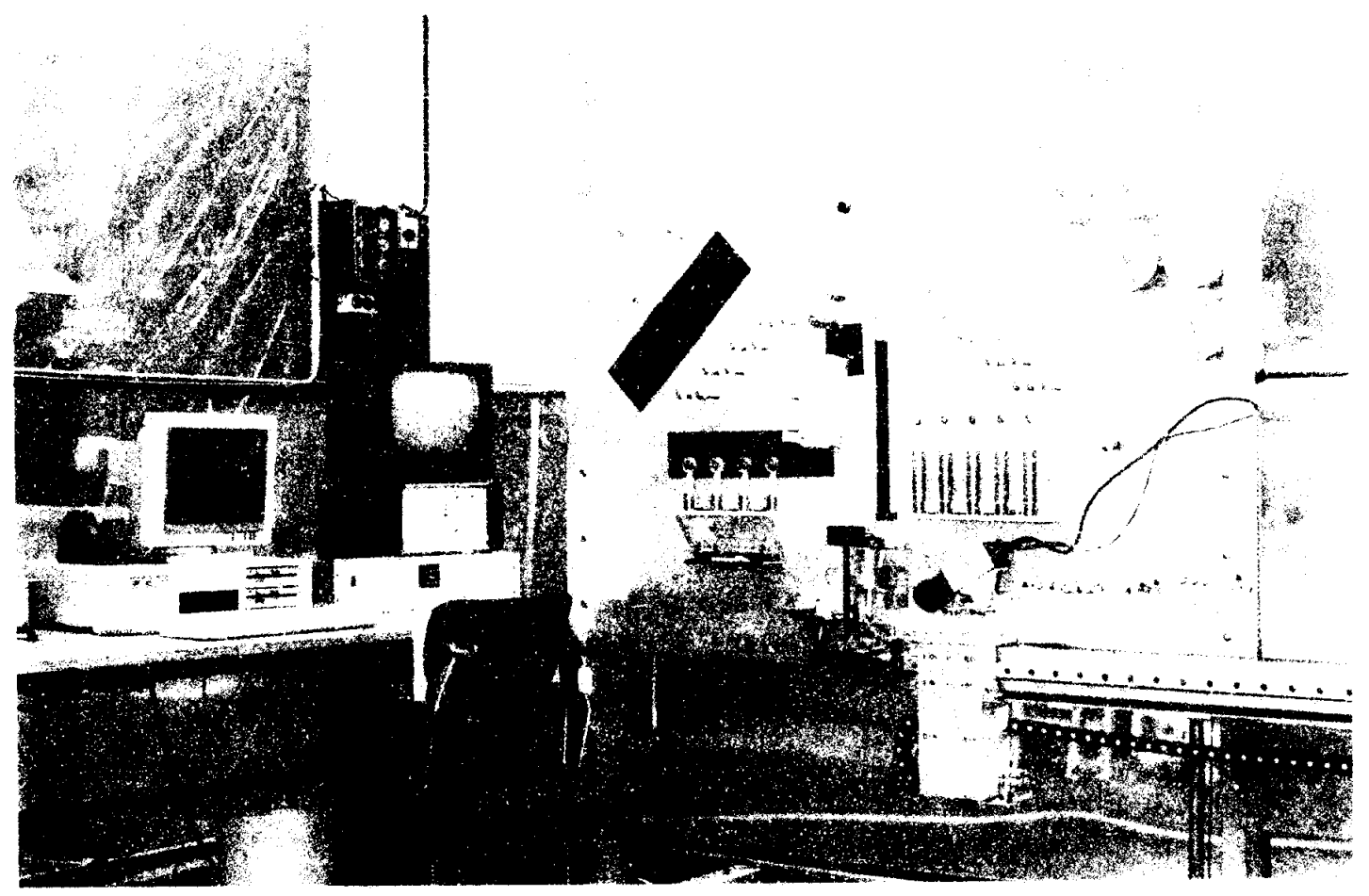

Figure 2: Photo of the wiring machine

The machine serves to prepare as a whole a wire group ontside the chamber, by stretching the four signal and five field wires of the group simultaneously. At one end the wires are crimped pncumatically on netal cubes of a Cu Te alloy inserted into the pockets of a polycarbonate block. A detailed description of the wire fixation scheme is given in Ref. 3. The block and wires are then puled through the appropriate length by a computer controlied step moior. Just before reaching the final setting, depending on the cell length required, the motor stops and magnetic brakes block the wire reels. The fixation block is then slowly moved to the final position, while, as shown in Figs. 2 and 3, a series of guide rollers serve to apply tension to the signal and field wires through the lifting of two sets of weights. The cther end of the wires is subsequently crimped on the CurTe pieces of two fixation blocks, which are then separated by cutting the wires between them. The second block stays on the machine to provide the start for stringing the next wire group, while the prepared group is transfered into the chamber manually by a team of two experienced operators. To avoid that the wires get angled with one another, (partial) tension is maintained while puiling the group though the chamber.

When installed in the namber, the wires are again stetched to the same length as on the wiring machine (the length of the drift cell) and should therefore have the design tension values. The tension of each wire is checked by measuring its oscillation frequency in an external magnetic field (see Sec, 4), thus providing immediate feedback to the operators regarding the quality of their work. Small departures of the 


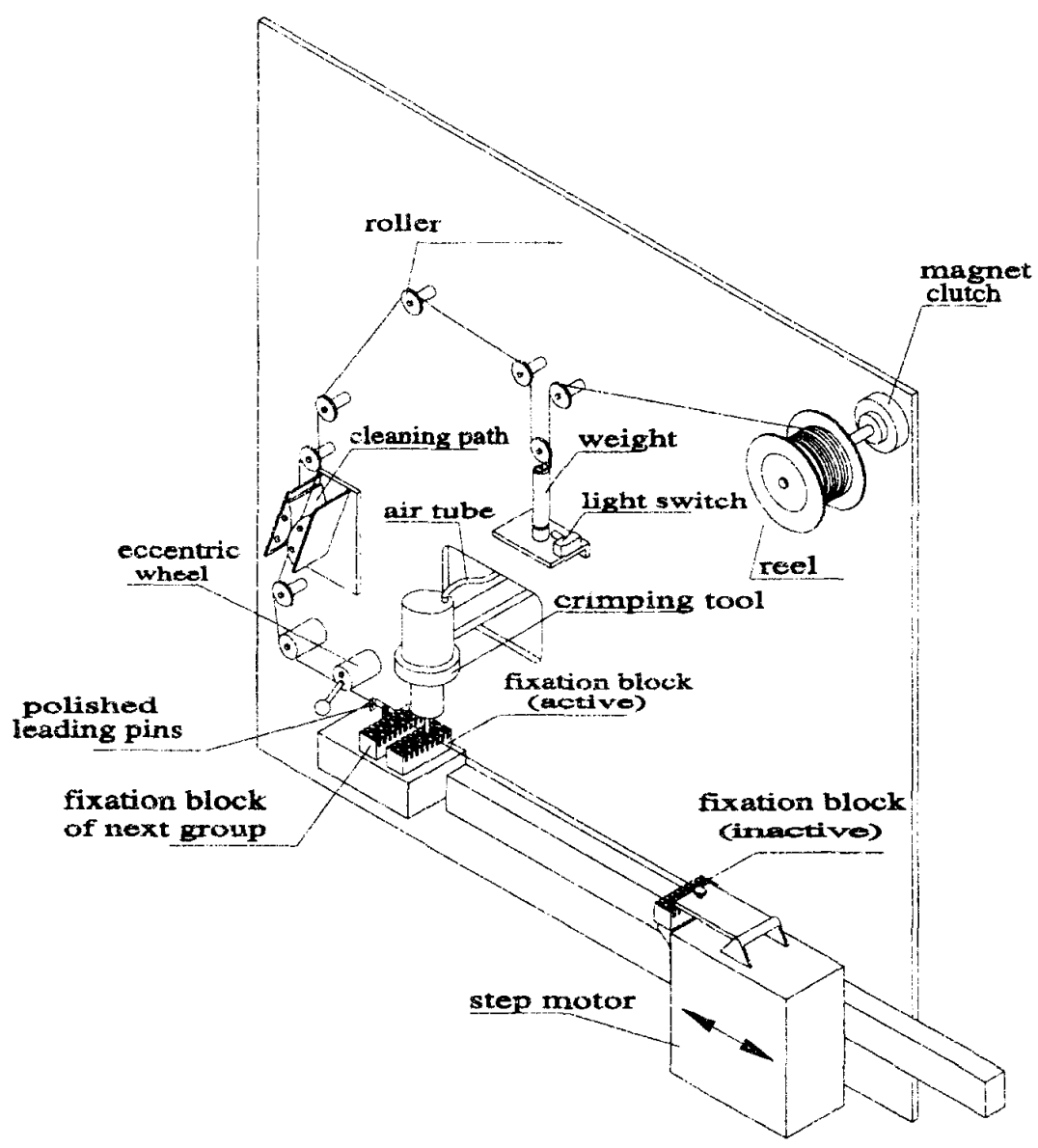

Figure 3: A schematic drawing of the wiring machine. The main features are shown exemplary for only one of all nine wires

tension from the intended values, such as may result from fluctuations in the position of the origin for the length measurement by the step motor or from small variations of chamber body dimensions away from the design values, can be compensated by changing and optimizing the programmed wire lengths (typically by $0.1-0.2 \mathrm{~mm}$ ). In case of large deviations from the intended values, the wire group can be replaced by stringing a new one. It is worth mentioning that such a replacement is needed only for $\approx 1 \%$ of the groups produced.

During placement of the wires inside the chamber their tension may temporarily exceed the design value. Since the latter is within $20 \%$ of the elastic limit, we performed a series of measurements to study the behaviour of the wires for values of the tension exceeding design. The results are summarized in Fig. 4(a,b) and demonstrate clearly the elastic behavior of both the signal and field wires in the neighborhood of the design tension values. 

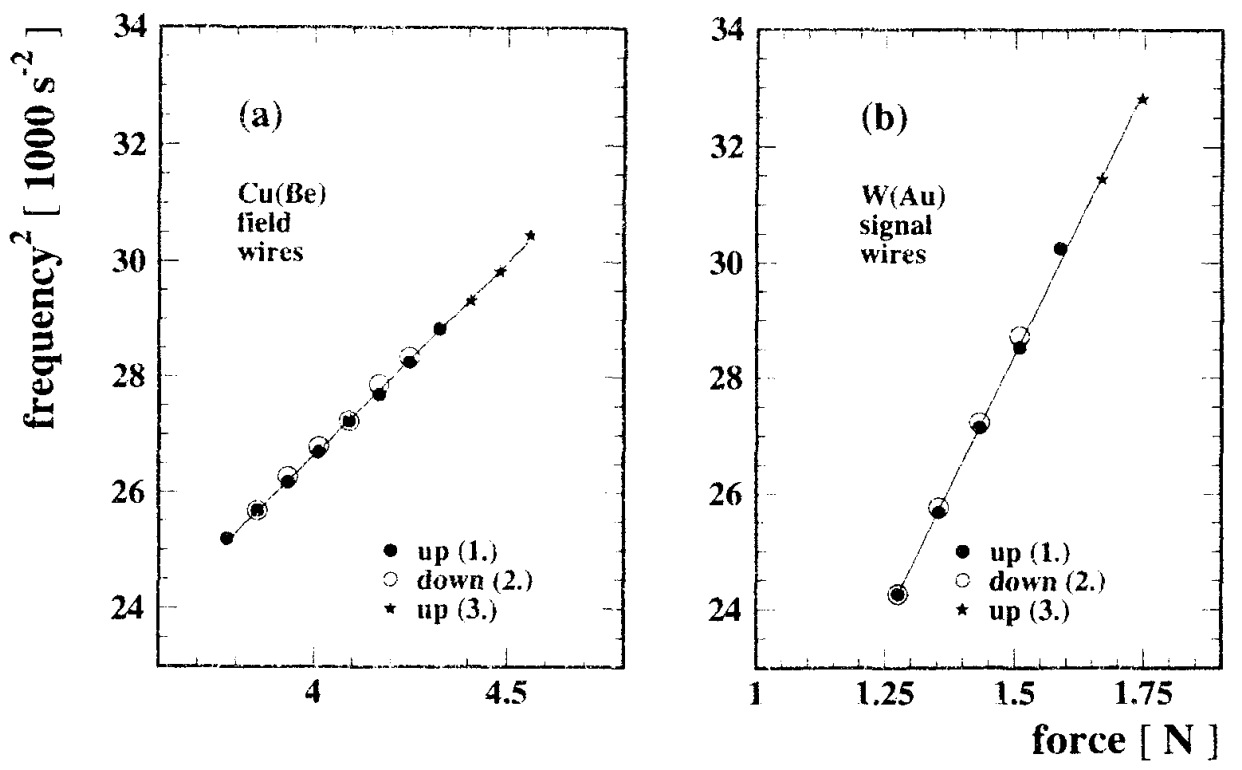

Figure 4: The square of the oscillation frequency $\left(\nu^{2}\right)$ versus the wire tension for (a) the field wires and (b) the signal wires. In this representation a linear relation is expected (see Eq. 4 in the text). The wires (with a length of $1 \mathrm{~m}$ ) were first stretched above the design tension (full circles). In a second step the tension was decreased back to the desigri value (open circles). Finally the tension was increased again until the wires broke (asterisks).

\section{Wire Tension Measurement}

We apply the electromagnetic method to obtain the mechanical tension of each stretched wire by measuring its fundamental ascillation frequency. A detailed description of the procedure and of the measuring apparatus has been given in Ref. [4]. Here we simply reiterate that the wire group is placed in the magnetic field generated by two bar magnets. The wires are oriented perpendicular to the field. A weakly damped oscillation is excited in each wire by feeding a short DC pulse into it. The oscillation frequency $\nu$ is the same as the induced $\mathrm{AC}$ frequency and is obtained by measuring the time $T_{10}$ for ten oscillations. The wire tension $\mathrm{F}$ is then obtained from ${ }^{4}$

$$
F=4 l_{c e l l}^{2} \nu^{2} \rho_{l}
$$

where $l_{\text {cell }}$ is the design value of the drift cell length.

The whole measurement process is controlled by a computer, which is also used to record and display the data.

As already mentioned, the agreement between measured and intended values of $F$ provides an indicator of the quality of the wire stringing and crimping. The overall

\footnotetext{
${ }^{4}$ The systematic error resulting from neglecting the weak damping of the cscillation in calculating
} $\mathrm{F}$ is less than $0.06 \cdot 10^{-3}$. 
uncertainty of the measurement is given by ${ }^{5}$

$$
\frac{\Delta F}{F}=\sqrt{4\left(\frac{\Delta \nu}{\nu}\right)^{2}+\left(\frac{\Delta \rho_{l}}{\rho_{l}}\right)^{2}}
$$

In our setup, the duration $T_{10}$ of ten oscillation periods $\mathrm{T}$ is measured five times with a precision $\Delta T_{1 i}<0.2 \mathrm{msec}[4]$. Thus the oscillation frequency is measured with an error

$$
\left.\frac{\Delta \nu}{\nu}=\frac{\Delta T}{T} \approx \frac{1}{\sqrt{5}} \frac{\Delta T_{10}}{T_{10}} \text { (largest for the shortest wires : } 0.3 \%\right) .
$$

The linear density $\rho_{l}$ was checked by (a) applying a well known weight to a vertically hanging wire and using the electromagnetic method described above to measure the fundamental resonant frequency of the wire (calibration method), and (b) weighing a piece of wire of known length 1 . Differences of up to $5 \%$ were found between individual rolls of wire. The main error in the determination of the tension would thus result from the uncertainty of the material constant $p_{l}$, exceeding the prescribed tolerance of $\pm 4 \%$ for the wire tension.

To avoid the large fluctuations in the knowledge of $\rho_{l}$, we used method (a) above to determine the linear density of each roll in the production process with an accuracy $\frac{\Delta \rho_{i}}{\rho_{l}}=0.4 \%$. Table 3 lists the different sources of error in the tension measurement and the overall precision achieved.

\begin{tabular}{|l|c|c|c|c|}
\hline wire length & $\frac{\Delta T_{10}}{T_{12}}\left[10^{-3}\right]$ & $\frac{\Delta \nu}{\nu}\left[10^{-3}\right]$ & $\frac{\Delta \beta_{1}}{\rho^{2}}\left[10^{-3}\right]$ & $\frac{\Delta F}{F}\left[10^{-3}\right]$ \\
\hline $473 \mathrm{~mm}$ & 6.4 & 2.9 & 4.0 & 7.0 \\
$2827 \mathrm{~mm}$ & 1.1 & 0.5 & 4.0 & 4.1 \\
\hline
\end{tabular}

Table 3: Errors in measurement of wire tension

\section{Results}

Typical distributions of the tension measured for field and signal wires are shown in Fig. 5(a,b), where the deviations from the intended values of $3.78 \mathrm{~N}$ and $1.28 \mathrm{~N}$, respectively, are plotted. Most of the measured values lie within the stipulated limits of $\pm 4 \%$. As established by the tests, whose results are summarized in Fig. 4, the positive deviations exceeding $4 \%$ are not critical, since the wires maintain their elastic behaviour for values of the tension well beyond the intended ones. Negative deviations are important only for the long wires (to avoid excessive sagging). As seen in Fig. 5, such deviations are within the requested tolerance for all long wires.

For each of the first 25 chambers wired in Aachen, Fig. 6(a,b) shows the average of the tension obtained for field and signal wires, respectively. The effect of the feedback provided by the tension measurement to the wire stringing operators is

\footnotetext{
${ }^{5}$ Given the chamber dimensions, possible deviations of the cell length from the design value $(0.1$ $-0.2 \mathrm{~mm}$ ) have a negligible effect on computing the wire tension from the measured oscillation frequency (Eq. 4).
} 

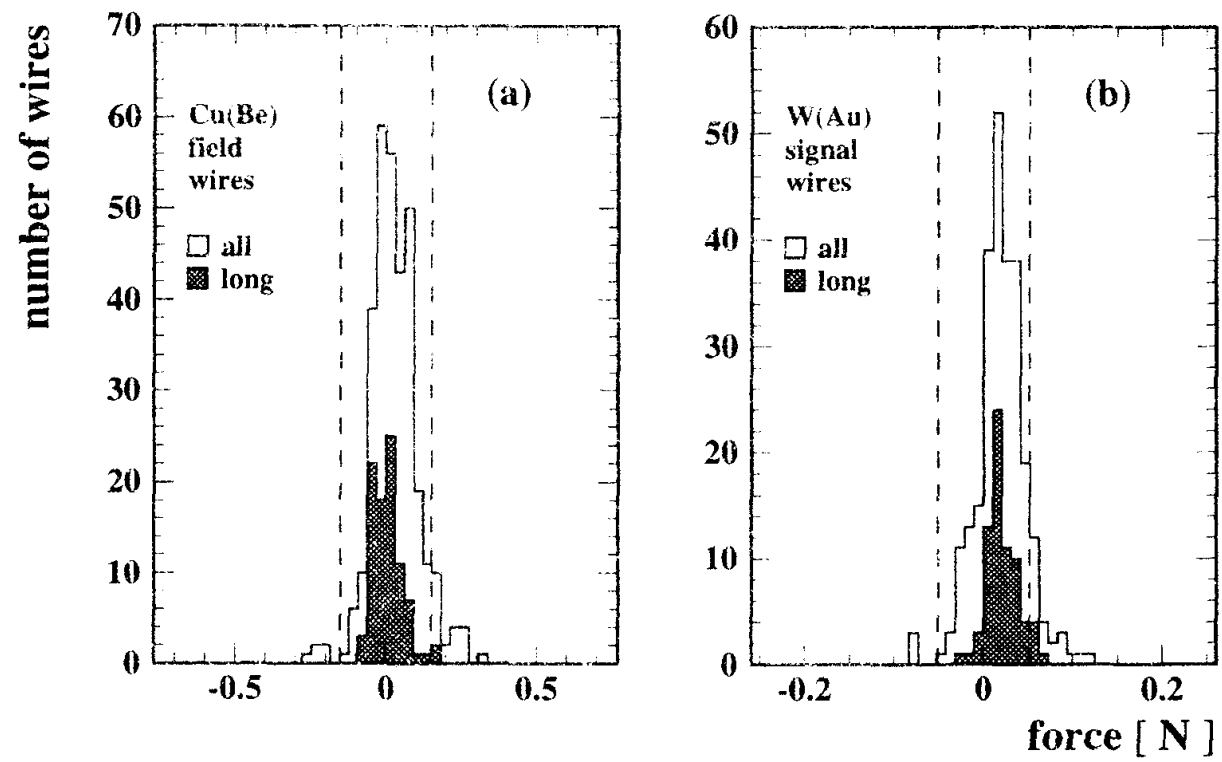

Figure 5: Deviation of wire tension from the design values for (a) field and (b) signal wires. Shadowed is the distribution for the critical long wires $(2826.9 \mathrm{~mm})$. The dashed lines show the design tolerance of $\pm 4 \%$.

obvious: the first chamber was clearly under-tensioned, an overcorrection cccurred for the second chamber, and then the operators settled to more or less constant values with slight variations. As indicated by the error bars in Fig. 6, within one chamber the r.m.s. of the tension is typically of the order of $2.5 \%$.

Measurements of the tension as a function of the time elapsed after wiring revealed a slow relaxation process, which, as noted above, is dangerous only for the long wires because of the sagging problem. To test the long time stability of the tension and to check for possible alterations due to transportation of the chamber, we repeatedly measured all wires of a particular chamber (nr. 2 in Tab. 4) over a period of 70 days following its wiring. Between the second and third measurements, the chamber, mounted on shock absorber, was loaded onto a truck and driven around for several hours. Fig. $7(a, b)$ displays the time development of the average value of the tension for the critical long wires. The measurements, corrected for temperature effects, show a similar behaviour for field and signal wires: After an initial decrease of $\approx 3 \%$ over a period of $\approx 15$ days, very little additional change is observed (see also Table 4 ), while transportation of the chamber appears to have had no significant effect. In particular, very similar values (open circle in Fig. 7) were obtained sixty days after wiring for a comparison chamber (nr. 5 in Tab. 4), which was kept in the laboratory and was not subjected to transport.

In addition, for four other chambers that were sent to Zürich, the wire tension was measured immediately after wiring in Aachen and after their arrival in Zürich. The results for the ling wires (not corrected for temperature effects) are summarized in Table 4. They show a decrease in tension similar to that obtained for chambers nr. 2 and 5 in the case of the signal wires, but a somewhat smaller relaxation of the field wires. 

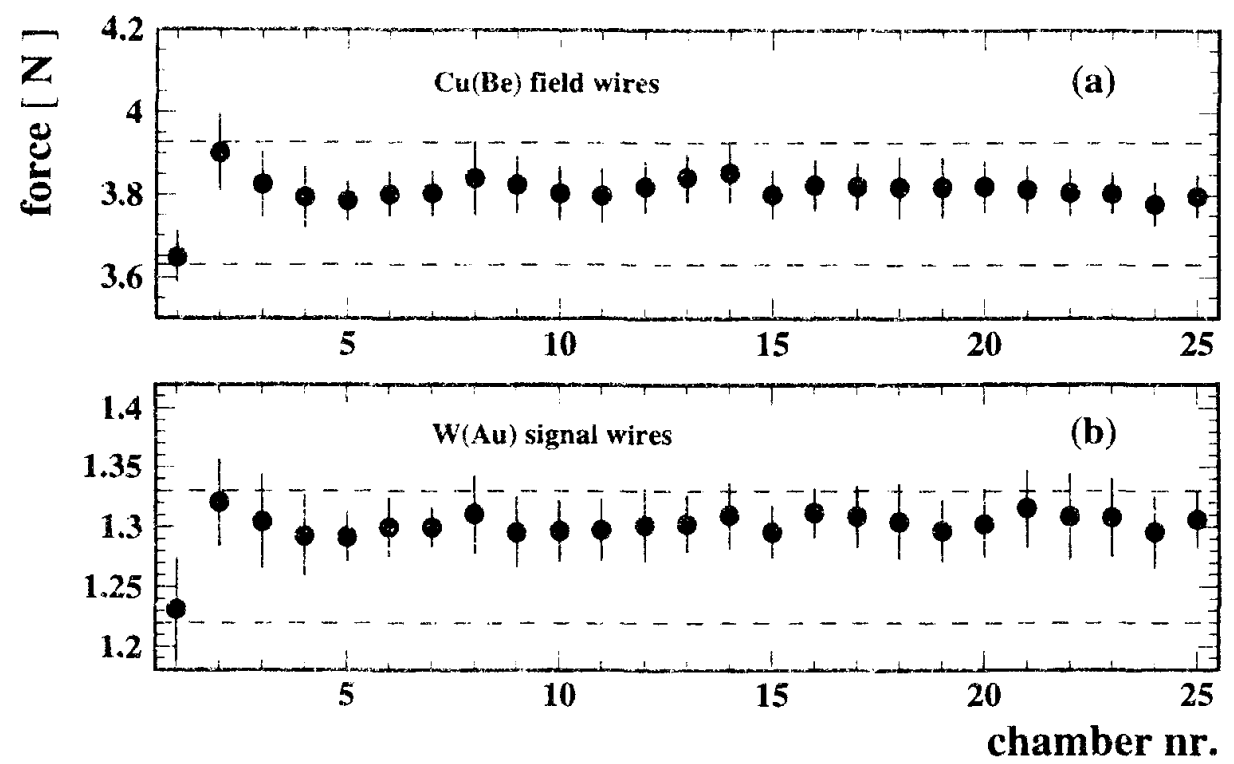

Figure 6: Avetage value of the tension for each of the first 25 chambers wired in Aachen for (a) the field wires and (b) the signal wires. The error bars indicate the standard deviation within one chamber. Mean and standard deviation are obtained from a gaussian fit on the distributions shown in figure 5. The horizontal lines show the $\pm 4 \%$ tolerance limits.

\begin{tabular}{|l|r|r|r|}
\hline chamber & $\begin{array}{r}\mathrm{Cu}(\mathrm{Be}) \text { wires } \\
\frac{\Delta F}{F}[\%]\end{array}$ & $\begin{array}{r}\text { W(Au) wires } \\
\frac{\Delta F}{F}[\%]\end{array}$ & $\begin{array}{r}\text { days after } \\
\text { wiring }\end{array}$ \\
\hline nr. 2 & -1.5 & -1.7 & 9 \\
nr. 2 & -2.2 & -2.6 & 14 \\
nr. 2 & -3.1 & -2.9 & 42 \\
nr. 2 & -3.3 & -3.0 & 66 \\
\hline nr. 5 & -3.0 & -2.4 & 61 \\
\hline nr. 19 & -1.5 & -3.4 & 28 \\
\hline nr. 20 & -0.9 & -2.2 & 15 \\
\hline nr. 21 & -0.7 & -2.3 & 10 \\
\hline nr. 22 & -0.6 & -2.4 & 2 \\
\hline
\end{tabular}

Table 4: Decrease in tension in percent of the initial tension for the long critical wires $(l=2827 \mathrm{~mm})$. In case of chambers $n r .2$ and $n r .5$ the measurements were corrected for temperature effects.

To compensate for the effect of the relaxation, which cannot be quantitatively calculated, the wires have been stretched with tensions close to the upper limit of the design value (see Fig. 6). 

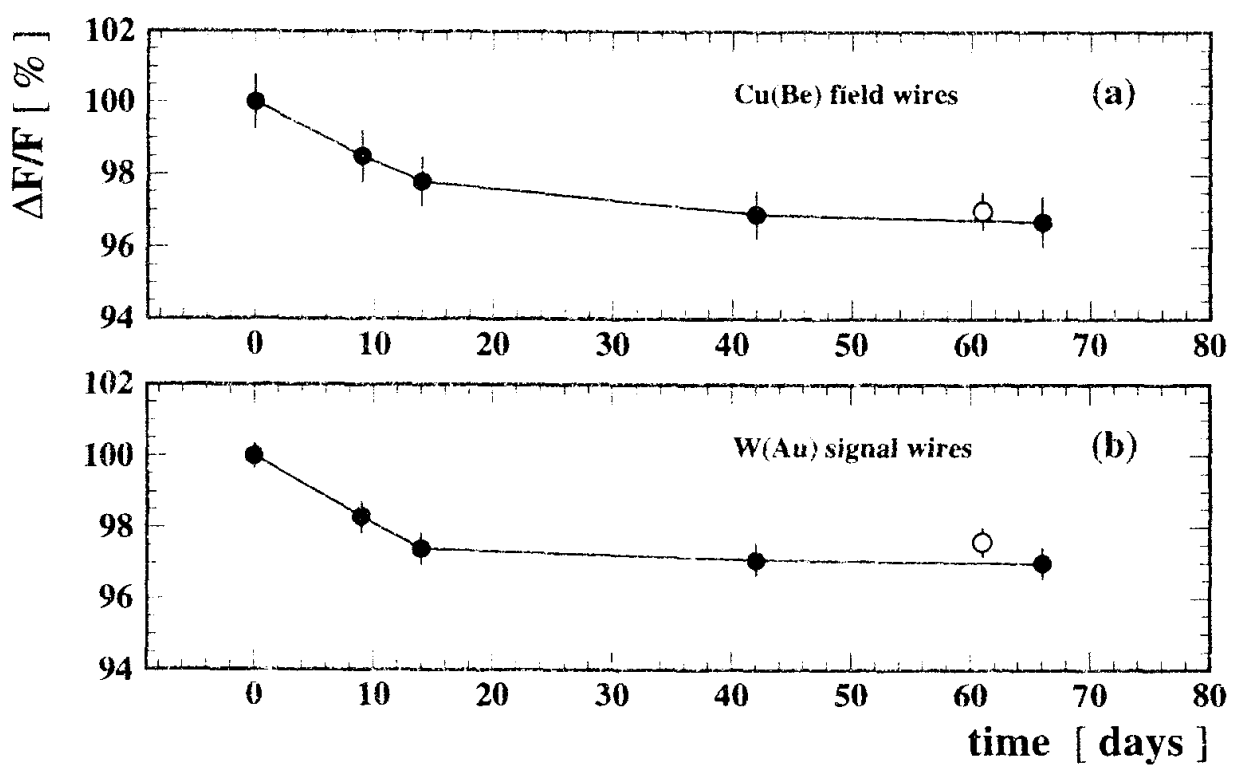

Figure 7: Time development of the average value of the tension of the long wires [(a) field wires,(b) signal wires], normalized to an initial value of $100 \%$. The solid circles correspond to the chamber subjected to the transportation test mentioned in the text (performed between the second and third measurements), while the open circle represents a comparison chamber (not transported).

\section{Conclusion}

We have developed a computer-controlled machine for the mass production stretching of the wires of the L3 forward/backward muon chambers within the stringent limits imposed on the wire tension: $1.28 \mathrm{~N} \pm 4 \%$ and $3.78 \mathrm{~N} \pm 4 \%$, respectively, for the goldplated Tungsten signal wires ( $30 \mu m$ diameter) and for the Copper-Beryllium field wires ( $78 \mu \mathrm{m}$ diameter).

The four signal and five field wires forming the wire group of one drift cell are all strung simultaneously. After the group has been transferred into the chamber, another computer-controlled device provides an immediate precision check of the tension ( $\Delta F / F \leq 0.5 \%$ for the longest wires), measuring the oscillation frequency of each wire in an external magnetic field. Large deviations from the design values can only be corrected by replacing the whole wire group, while small but systematic deviations can be compensated by small changes (typically $0.1-0.2 \mathrm{~mm}$ ) of the programmed values of the wire lengths.

We observed a relaxation of the tension by $\approx 3 \%$ within a period of $\approx 15$ days after wiring, but little subsequent change, and corrected for this initial decrease by stretching the wires with a tension close to the upper limit of the design values. Finally, we checked that transporting the chambers did not have any significant effect on the wire tension values. 


\section{References}

[1] L3 Collaboration, B. Adeva et al., Nucl. Instr. and Meth. A.289 (1990) 35.

[2] U. Becker, Large Area and Muon Detectors, in Instrumentation in High Energy Physics (Ed. F. Sauli), World Scientific Publishing Co., Singapore, 1992.

[3] R. Bock et al., Nucl. Instr. and Meth. A336 (1993) 128, and RWTH Aachen Report, PITHA 93/25 (1993)

[4] G. Braun et al., A Computer Controlled Device for Measuring the Wire Tension in Drift and Proportional Chambers, in preparation. 
